\title{
Volumetric modulated arc therapy for treatment of solid tumors: current insights
}

\author{
This article was published in the following Dove Press journal: \\ OncoTargets and Therapy \\ 26 July 2017 \\ Number of times this article has been viewed
}

\author{
Gabriella Macchia' \\ Francesco Deodato' \\ Savino Cilla ${ }^{2}$ \\ Silvia Cammelli ${ }^{3}$ \\ Alessandra Guido ${ }^{3}$ \\ Martina Ferioli ${ }^{3}$ \\ Giambattista Siepe 3 \\ Vincenzo Valentini ${ }^{4}$ \\ Alessio Giuseppe \\ Morganti ${ }^{3, *}$ \\ Gabriella Ferrandina $5,6, *$ \\ 'Radiation Oncology Unit, ${ }^{2}$ Medical \\ Physics Unit, "Giovanni Paolo II" \\ Foundation, Catholic University of the \\ Sacred Heart, Campobasso, ${ }^{3}$ Radiation \\ Oncology Unit, Department of \\ Experimental, Diagnostic and Specialty \\ Medicine, University of Bologna, \\ S. Orsola-Malpighi Hospital, Bologna, \\ ${ }^{4}$ Department of Radiation Oncology, \\ Catholic University of the Sacred \\ Heart, Rome, ${ }^{5}$ Department of \\ Obstetrics and Gynecology, Catholic \\ University of the Sacred Heart, Rome, \\ ${ }^{6}$ Department of Health Sciences \\ and Medicine, University of Molise, \\ Campobasso, Italy
}

*These authors contributed equally to this work
Correspondence: Francesco Deodato Radiation Oncology Unit, "Giovanni Paolo II" Foundation, Catholic University of the Sacred Heart, Largo Gemelli I, 86100 , Campobasso, Italy

Tel +390874 312259

Fax +390874 312 720

Email fdeodato@rm.unicatt.it
Aim: This article discusses the current use of volumetric modulated arc therapy (VMAT) techniques in clinical practice and reviews the available data from clinical outcome studies in different clinical settings. An overview of available literature about clinical outcomes with VMAT stereotactic/radiosurgical treatment is also reported.

Materials and methods: All published manuscripts reporting the use of VMAT in a clinical setting from 2009 to November 2016 were identified. The search was carried out in December 2016 using the National Library of Medicine (PubMed/Medline). The following words were searched: "volumetric arc therapy"[All Fields] OR "vmat"[All Fields] OR "rapidarc"[All Fields], AND "radiotherapy"[All Fields] AND "Clinical Trial”[All Fields].

Results: Overall, 37 studies (21 prospective and 16 retrospective) fulfilling inclusion criteria and thus included in the review evaluated 2,029 patients treated with VMAT; of these patients, $\sim 30.8 \%$ had genitourinary (GU) tumors (81\% prostate, $19 \%$ endometrial), $26.2 \%$ head-and-neck cancer (H\&NC), 13.9\% oligometastases, $11.2 \%$ had anorectal cancer, $10.6 \%$ thoracic neoplasms ( $81 \%$ breast, 19\% lung), and 7.0\% brain metastases (BMs). Six different clinical scenarios for VMAT use were identified: 1) BMs, 2) H\&NC, 3) thoracic neoplasms, 4) GU cancer, 5) anorectal tumor, and 6) stereotactic body radiation therapy (SBRT) performed by VMAT technique in the oligometastatic patient setting.

Conclusion: The literature addressing the clinical appropriateness of VMAT is scarce. Current literature suggests that VMAT, especially when used as simultaneous integrated boost or SBRT strategy, is an effective safe modality for all cancer types.

Keywords: VMAT, RapidArc, clinical trial, review, radiosurgery, stereotactic, simultaneous integrated boost

\section{Introduction}

Since the introduction of intensity-modulated radiotherapy (IMRT) in clinical routine in the late 1990s, a very fast growth has characterized radiotherapy (RT) technology offering newer technologies and techniques to radiation oncologists. Indeed, IMRT, ${ }^{1}$ helical tomotherapy, ${ }^{2}$ intensity-modulated arc therapy (IMAT), ${ }^{3}$ and volumetric modulated arc therapy (VMAT) ${ }^{4}$ enable better radiation dose conformality to the target volume compared to three-dimensional conformal radiotherapy (3D-CRT).

Moreover, these techniques, which underlie various complex computer-based optimization algorithms, allowed the delivery of nonuniform radiation beam intensities in order to obtain highly conformal dose distributions, thus potentially resulting in RT dose escalation to the target with an improved cancer control. Moreover, they allowed the delivery of simultaneous integrated boost (SIB), a technique that permits treating of several volumes safely with different dose prescriptions, thus leading to reduction of the dose to the surrounding radiation-sensitive normal tissues and improvement of the toxicity profile. 
In this context, VMAT represents the newest RT technique and can provide additional advantages, such as reduced treatment delivery time compared with conventional static field IMRT. In fact, in VMAT delivery, field shapes, dose rate, and gantry rotation speed can simultaneously vary. These additional degrees of freedom increased the capability of beam intensity modulation with respect to IMAT. Based on Otto's VMAT algorithm, ${ }^{4}$ Varian (Palo Alto, CA, USA) implemented the single-arc form of IMAT and named the system RapidArcTM ${ }^{\circledR}$. Elekta (Stockholm, Sweden) and Philips (Amsterdam, the Netherlands) also released their rotational IMRT solutions, named VMAT ${ }^{\circledR}$ and $\mathrm{SmartArcTM}^{\circledR}$, respectively. Since the clinical implementation of these different rotational forms of IMRT by different vendors, the feasibility of applying this novel delivery technique to different cancer sites has been widely explored. Theoretical investigation and a very large number of treatment planning studies have extensively addressed the differences among VMAT, 3D-CRT, IMRT, and helical tomotherapy. ${ }^{5-15}$

In the last decade, technological improvements in setup, imaging, accuracy in dose delivery, and the ability to compensate for respiratory motion have led to a widespread clinical implementation of stereotactic body radiation therapy (SBRT), also named as stereotactic ablative radiotherapy (SABR). SBRT refers to the delivery of large focused doses over a limited number of fractions to tumor sites, in order to obtain the highest biological effective dose. Because of its rotational nature and its fast delivery timing, VMAT has been immediately recognized as an ideal technique for SBRT that requires steep dose gradients, high precision, and reduction of treatment time, thus minimizing the risk of intrafraction setup deviations or organ motion. ${ }^{16}$ In this context, a few dosimetric and feasibility studies have confirmed high-dose conformity and fast delivery time in several types of malignancies. ${ }^{17-23}$

The clinical worldwide use of VMAT is significantly increasing; despite that, the majority of published data are currently confined to planning and feasibility studies. ${ }^{5-15}$ Results relative to toxicity and clinical outcome are emerging, but still sparse.

This article aims to discuss the current use of VMAT techniques in clinical practice and review the available data from clinical outcome studies in different clinical settings including brain metastases (BMs), head and neck primary neoplasms, thoracic tumors, lower gastrointestinal (GI), and genitourinary (GU) cancers. Moreover, an overview of the available literature about clinical outcomes with VMAT stereotactic/radiosurgical treatment is reported.

\section{Materials and methods}

\section{Search strategy}

All published manuscripts reporting the use of VMAT in a clinical setting from 2009 (ie, 1 year after VMAT technique implementation) to November 2016 were identified. The search was carried out in December 2016 using the National Library of Medicine (PubMed/Medline). The following words were searched: "volumetric arc therapy"[All Fields] OR "vmat"[All Fields] OR "rapidarc"[All Fields], AND "radiotherapy”[All Fields] AND “Clinical Trial”[All Fields]. In order to identify other possible studies of interest, this process was supplemented by manual examination of reference lists for the available review articles ${ }^{24,25}$ or clinical trial papers.

\section{Selection criteria}

Eligible studies met the following criteria: published paper investigating an adult population with any cancer treated with VMAT, regardless of clinical indication or setting (ie, exclusive, neoadjuvant, adjuvant, and palliative). Additional inclusion criteria were studies, 1) with oncological or toxicity outcomes from VMAT, and 2) published in the English language. Excluded publications were, 1) dosimetric or feasibility studies without clinical data; 2) reviews, editorials, case reports, conference abstracts, and letters to editor; 3) repeated publications by the same institution (ie, analysis was restricted to the most recent or the most comprehensive one); 4) papers reporting comparison between techniques from which it was not possible to extract and separately evaluate the outcome data of 1 arm over the other.

The title, abstract, and keywords of the identified articles were independently analyzed by a pool of researchers (GS, MF, SC, and AG) and disagreement was resolved by 2 supervisors' opinions (GM and FD); thereafter, papers not suitable for analysis were excluded as mutually agreed.

Potentially eligible studies were retrieved and a full-text evaluation was performed as to whether they satisfied the inclusion and exclusion criteria.

\section{Data collection and analysis}

The following data were collected by at least 2 researchers: author's name and year of publication, study design, clinical setting, number of treated patients, tumor site, VMAT dose, fractionation and technique, image guidance (IG) availability, acute and late toxicity, follow-up time, clinical finding, local control, disease-free survival (DFS), and overall survival (OS). A descriptive analysis was used for the data report. The results are stratified by anatomic site to aid in the interpretation. 


\section{Results}

According to the search strategy, we were able to identify 116 citations, 97 identified through database searching and 19 identified through other sources (review articles). Based on the title and abstract, 72 publications were excluded because they involved dosimetric issues $(\mathrm{n}=62)$, or irrelevant topics $(\mathrm{n}=10)$, leading to 42 full-text articles selected for full inspection. Subsequently, 7 papers were excluded since they reported subset analyses of already selected papers, a case series, and an early halted trial, thus leaving 37 studies (21 prospective and 16 retrospective) fulfilling inclusion criteria and therefore included in the final review (Figure 1).

Overall, these studies evaluated 2,029 patients treated with VMAT; of these patients, $\sim 30.8 \%$ had GU tumors ( $81 \%$ prostate, $19 \%$ endometrial), $26.2 \%$ head-and-neck cancer (H\&NC), $13.9 \%$ oligometastases, $11.2 \%$ anorectal cancer, $10.6 \%$ thoracic neoplasms ( $81 \%$ breast, $19 \%$ lung), and $7.0 \%$ BMs.

Six different clinical scenarios for VMAT use were identified: 1) BMs, 2) H\&NC, 3) thoracic neoplasms, 4) GU cancer, 5) anorectal tumor, and 6) SBRT performed by VMAT technique in the oligometastatic patient setting.

For the sake of clarity, we will present and discuss data, site by site.

\section{BMs: exclusive, adjuvant, and prophylactic settings}

BMs are the most common brain tumors in adults and occur in $20 \%-40 \%$ of patients with cancer; ${ }^{26} \mathrm{BMs}$ often cause morbidity and mortality because of effect of the mass or brain site-related symptoms. ${ }^{27-29}$ The use of a sophisticated technique, such as the VMAT, in this setting is driven by the aim of reducing the irradiation of healthy brain, potentially allowing dose escalation (Table 1). ${ }^{30-33}$ In this context, 3 studies have investigated the clinical efficacy of VMAT-SIB in different clinical settings including exclusive treatment in the vast majority of cases. ${ }^{30-32}$ It has to be acknowledged that the VMAT-SIB approaches diverged in terms of total dose, dose per fraction, and timing, thus sustaining a different toxicity profile. In particular, the use of high dose per fraction resulted in higher radionecrosis. ${ }^{32}$ Overall, the available studies confirmed that BMs can be successfully managed with a satisfying clinical outcome; indeed, despite the heterogeneity of follow-up length as well as clinical end points, the rate of local control was $\sim 80 \%$. In this context, the optimization of the therapeutic window is clinically relevant since the rate of impairment of neurocognitive functions is not negligible ${ }^{32}$ and, more importantly, leads to a large amount of social and family sequelae. Prospective assessment of neurocognitive functions before and after hippocampus-sparing whole-brain radiotherapy (WBRT) has shown that adherence to the reference constraints guarantees preservation of neuro-cognitive functions and verbal memory. ${ }^{33}$ The only caveat remains the deep brain metastases (basal ganglia and thalamus) where the risk of radionecrosis resulted unacceptable at the suggested doses. ${ }^{32}$

\section{H\&NC: exclusive and adjuvant settings}

$\mathrm{H} \& \mathrm{NC}$ accounts for $6 \%$ of all malignancies, and almost half of patients present with a locally advanced stage due to an

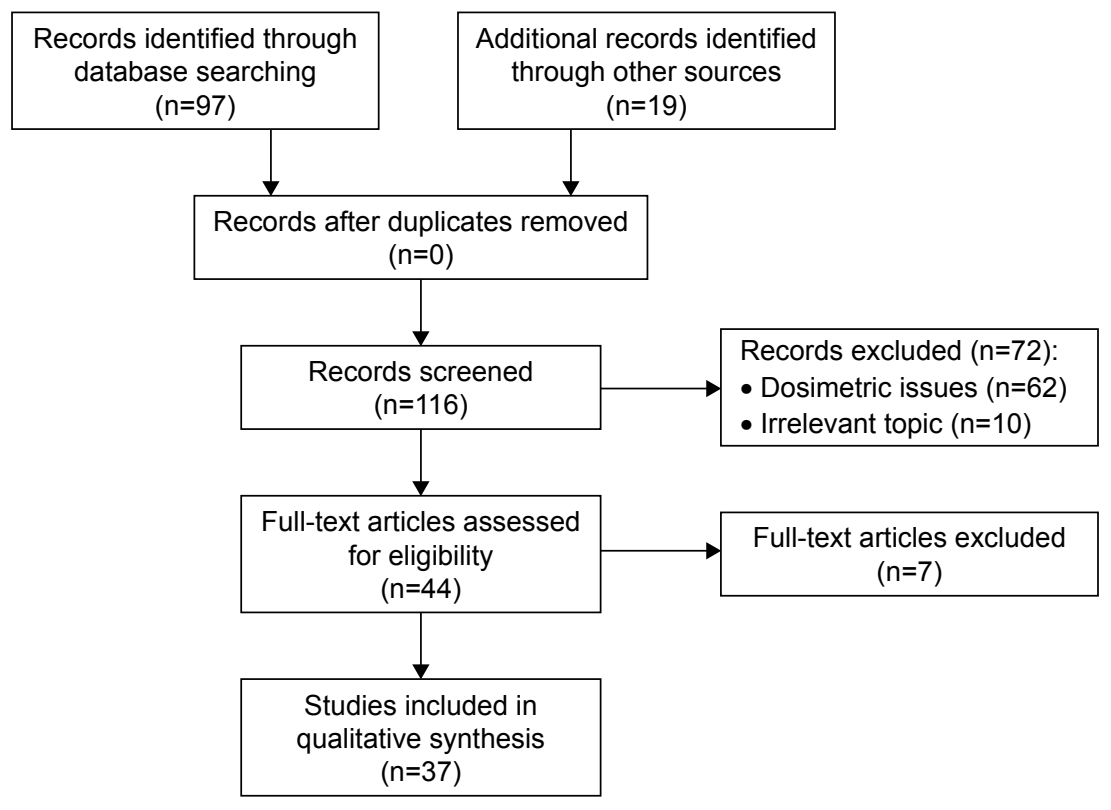

Figure I Flow chart - study selection diagram. 


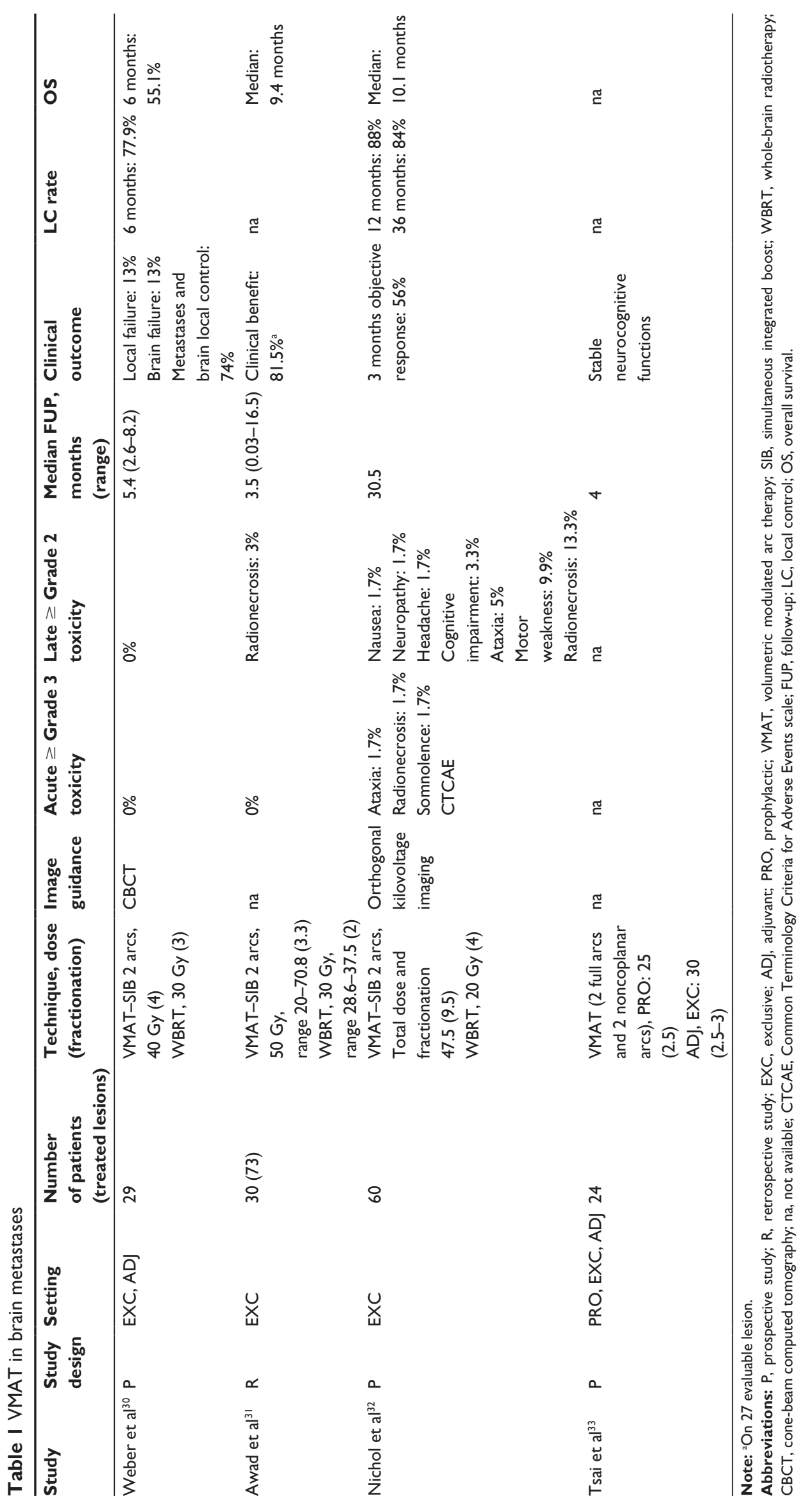


aggressive phenotype and rapid growth because of the rich lymphatic supply of this region. ${ }^{34} \mathrm{RT}$ for $\mathrm{H} \& \mathrm{NC}$ can be challenging due to the complex anatomy, with tumors often located within close proximity to critical structures (spinal cord, salivary glands, eyes, and dysphagia-related structures) which can limit radiation dose. RT is an important treatment modality in these tumors as it offers an alternative treatment option to surgical resection which can cause unacceptable cosmetic disfigurement and functional impairment. ${ }^{35,36} \mathrm{In}$ the last decade, IMRT has gradually assumed an important role in the management of such diseases because it enables delivery of highly conformal dose distributions and increases the therapeutic ratio as target volumes are often large and concave around nearby critical normal tissues. ${ }^{37-39}$ As shown in the randomized PARSPORT trial (ISRCTN48243537), IMRT can reduce late toxicity parameters, such as xerostomia, by increasing sparing of the parotid glands ${ }^{40}$ However, it has several drawbacks such as complexity of treatment planning and delivery requiring extensive quality assurance, prolonged beam delivery time which may worsen the accuracy of treatment because of increased intrafractional patient motion, and reduced patient throughput with subsequent longer waiting lists. Another issue of concern is the increased number of monitor units (MUs) required for IMRT, which could increase the number of secondary malignancies after curative treatment. ${ }^{41}$ In this context, VMAT represents a valid strategy to overcome the above-cited IMRT drawbacks. Indeed, planning studies have reported that VMAT plan quality is at least comparable to standard IMRT, with shorter planning and delivery time, and less MUs. ${ }^{7,8,14,42}$

A few retrospective clinical studies have investigated the impact of VMAT in H\&NC, totaling $~ 533$ patients ${ }^{43-48}$ (Table 2); also in this setting, the dual-arc-VMAT-SIB technique was the dosimetric treatment of choice. Prophylactic treatment of lymph nodes was performed with doses between 40 Gy and 57.75 Gy according to different protocols, with fractionation ranging from 1.3 to 1.8 Gy per fraction. The visible disease documented by imaging and/or physical examination received doses up to $75 \mathrm{~Gy}$, with dose fraction ranging between 2 and $2.4 \mathrm{~Gy}$. Three of the 6 studies reported the use of IG ( $\mathrm{kV}$ or MV) during delivery. ${ }^{43,44,47} \mathrm{~A}$ limitation present in all studies was the enrollment of mixed histotypes, except for the Guo et al study that reported the largest VMAT-SIB nasopharyngeal carcinoma series $(\mathrm{N}=205) .^{48}$ Toxicities $\geq$ Grade 3 were confluent mucositis (range $28 \%-49 \%$ ), cutaneous moist desquamation (14\%-38\%), dysphagia ( $7 \%-63 \%)$, and pain requiring opioid analgesia (49\%). Outcome data were provided by Scorsetti et al ${ }^{43}$ who

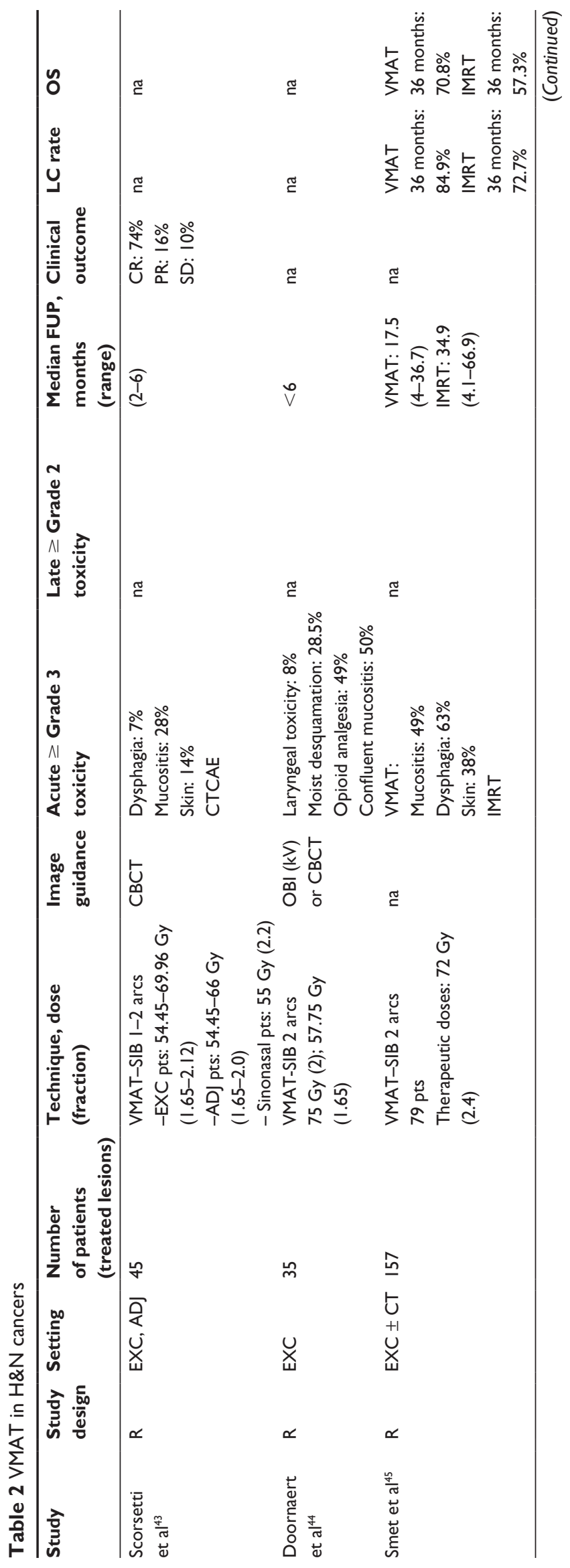




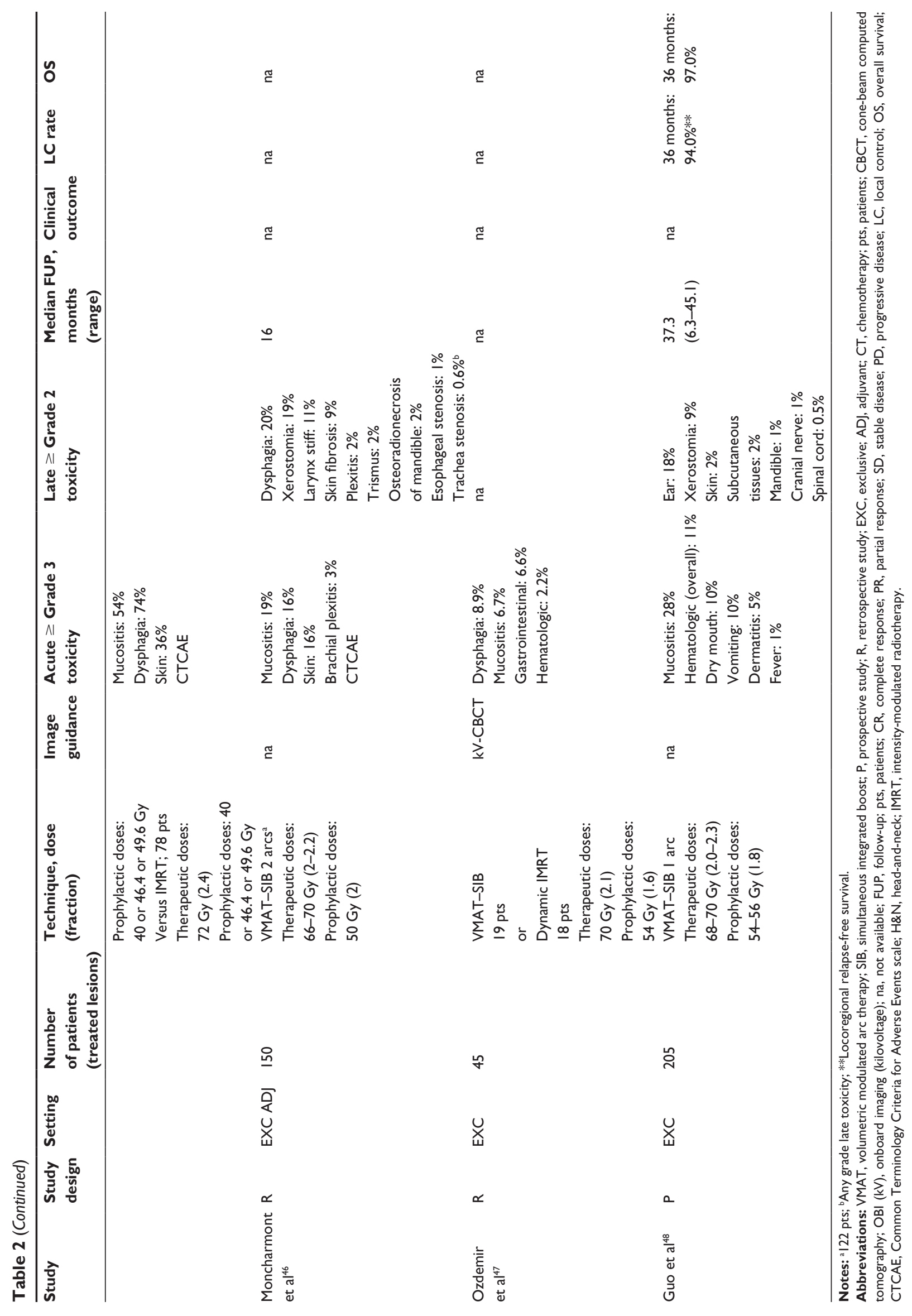


reported that clinical response to treatment was encouraging, by Smet et $\mathrm{al}^{45}$ who reported survival data favoring VMAT compared to IMRT, and by Guo et $\mathrm{al}^{48}$ who reported a 36-month locoregional relapse-free survival of $94 \%$ and a 36-month OS of $97.0 \%$ (Table 2).

In conclusion, preliminary results in locally advanced H\&NC treated with VMAT showed acceptable or favorable toxicity profile ${ }^{48}$ even using concomitant chemotherapy. Duration of follow-up within the available studies is heterogeneous; moreover, in the only study providing a comparison between the 2 techniques, follow-up duration was shorter in patients managed with VMAT compared to IMRT. ${ }^{45}$ Prospective trials with longer follow-up are needed to achieve reliable conclusions on this matter.

\section{Thoracic neoplasm: exclusive and adjuvant settings}

A few heterogeneous clinical studies have investigated the use of VMAT in thoracic neoplasm (Table 3); ${ }^{49-52}$ the rationale to use VMAT in this field sounds reasonable since the IMRT quality dose distributions can still be achieved, but in a shorter treatment time that could minimize the impact of intrafraction motion. ${ }^{53}$ Moreover, the convex hull lung tumor shape makes the delivery of VMAT advantageous and competitive with respect to conventional IMRT in terms of contralateral lung and spinal cord sparing. Concerns still remain regarding the increase of normal tissue volume receiving low-dose radiation in addition to the potential increased risk of secondary malignancy induction especially for breast disease. ${ }^{53}$

Scorsetti et $\mathrm{al}^{49}$ reported acute toxicity, initial outcome results, and therapeutic planning parameters in radiation treatment of advanced lung cancer (Stage III) with volumetric modulated arcs using RapidArc (RA). The earliest published study investigated the clinical outcome of 24 nonsmall cell lung cancer patients treated with VMAT: despite the large target volumes (gross tumor volume: $299 \pm 175 \mathrm{~cm}^{3}$, planning target volume: $818 \pm 206 \mathrm{~cm}^{3}$ ) and delivery of curative doses, no severe toxicity was recorded. Moreover, partial response and stabilization of disease were documented in $78 \%$ and $22 \%$ of cases, respectively, thus highlighting the therapeutic potential of this strategy which nonetheless requires further investigations. ${ }^{49}$

Another field of application of VMAT in the thoracic area is represented by pleural mesothelioma whose treatment after extrapleural pneumonectomy can include adjuvant irradiation. ${ }^{50}$ Based on the encouraging results obtained with adjuvant IMRT in terms of increased delivered doses and better local control compared to 3D-CRT technique, attention has been focused on the use of VMAT in the same clinical setting because of the possibility of reducing the contralateral lung dose volume receiving $\geq 5$ Gy (V5), or the mean lung dose thus resulting in a lower rate of severe pulmonary toxicity. Indeed, only 1 study has addressed this issue reporting a rate of acute Grade 3 pneumonitis in $20 \%$ of patients, a figure that favorably matches with previously reported data on IMRT. ${ }^{54,55}$

The rationale of VMAT in breast cancer adjuvant treatment concerns a specific subset of patients whose hearts are positioned close to the chest wall and who could not be adequately treated with a modified wide-tangent technique. In dosimetric studies, VMAT plans showed a statistically significant reduction in mean dose to the heart and ipsilateral (left) lung compared with IMRT ${ }^{56}$ A reduction in the volume of heart and lung receiving low doses (eg, 10 Gy and 5 Gy) may be important in the subgroup of patients who have received anthracycline-based chemotherapy and/or trastuzumab. The mean dose to the full contralateral breast was lower as well. ${ }^{56}$ Scorsetti et al reported the use of the 3-week VMAT-SIB course as adjuvant treatment after breast-conserving surgery; ${ }^{57}$ their Phase II trial showed that the technique is well tolerated and associated with optimal local control. ${ }^{52}$

To our knowledge, 2 studies analyzed the role of VMAT in the exclusive as well as adjuvant setting. ${ }^{51,52}$ In particular, in the larger prospective series by De Rose et al, VMATSIB (40.5 Gy on the whole breast, 48 Gy on surgical bed) provided a very favorable acute and late toxicity profile; ${ }^{52}$ with the limits inherent in the relative short follow-up, an optimal 2-year local control rate was documented.

A different clinical setting was investigated by Kim et al who delivered up to 50 Gy to the whole breast and up to $60-70$ Gy on tumor sites. ${ }^{51}$ As far as toxicity is concerned, late Grade $\geq 2$ was registered in only $3.2 \%$ of cases.

\section{GU cancer: exclusive and adjuvant settings}

The increasing incidence of prostate cancer (PC) and endometrial cancer (EC), as well as the favorable rate of curability and long survival, justifies the strenuous pursuit of healthy tissue toxicity reduction policy.

Indeed, treatment of pelvic lymph nodes as well as the primary tumor or tumor bed is indicated in high-risk PC and EC patients by international guidelines [www.NCCN. org]. The so-called horseshoe shape of the lymph node target, which includes the small intestine and rectum in its concavity, represents a condition particularly suitable to benefit from the intensity-modulated techniques and especially from VMAT. 


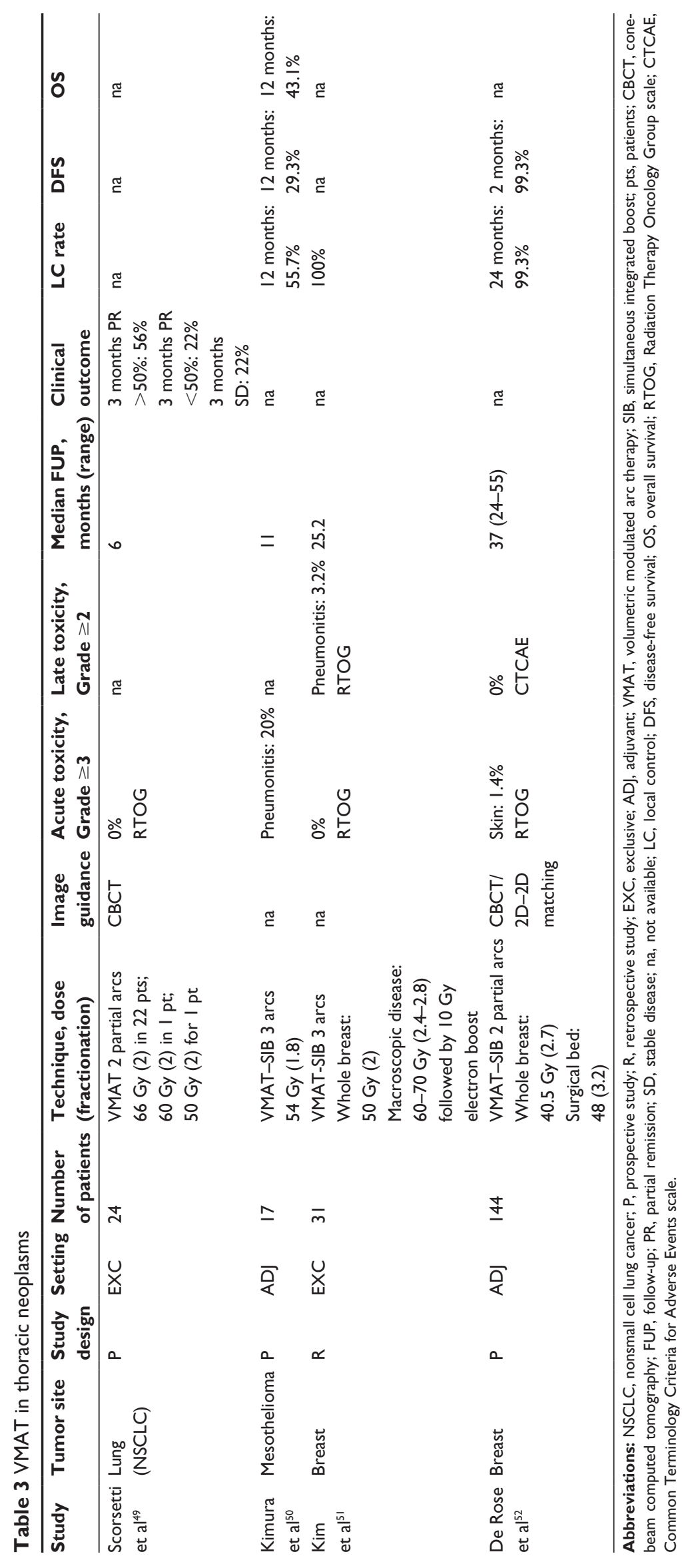


Concerning PC, dosimetric studies demonstrated a comparable or higher sparing to organs at risk (OARs) obtained with VMAT in comparison with the IMRT plans, especially by dual-arc technique that can obtain a superior conformity and homogeneity compared with single-arc plans. ${ }^{58-60}$ In addition, the better efficiency of VMAT delivery reduces the treatment time, making it an attractive solution for radiation oncologists. ${ }^{58-60}$

Eight clinical studies ( 5 prospective and 3 retrospective) have been reviewed in this study; ${ }^{61-68} \mathrm{PC}$ represents the disease on which VMAT has been employed since the beginning and most frequently.

Five studies addressed only exclusive setting, ${ }^{62,64-67}$ while the remaining studies investigated adjuvant setting ${ }^{63}$ or both. ${ }^{61,68}$ Most of the patients enrolled in these studies (including series between 23 and 113 patients) were treated mostly by VMAT-SIB dual-arc and triple-arc techniques, almost all using IG as kilovoltage $(\mathrm{kV})$ or cone-beam computed tomography (CBCT). It is important to mention the importance of IG during RT, for example, with CBCT. This enables patient positioning errors to be followed and corrected with high precision. In PC, where internal organ movement is a key point for patient positioning and target localization, ${ }^{69} \mathrm{IG}$ permits the safe delivery of higher doses per fraction. Pesce et al and Sveistrup et al treated only prostate volume, thus using single-arc technique up to $78 \mathrm{~Gy} / 2$ Gy fraction; ${ }^{61,65}$ both authors reported that IG-VMAT is a safe treatment for PC, with few and mild changes in urinary and GI symptoms after 1 year from RT completion. Sexual symptoms deteriorated during and after RT; however, Pesce et $\mathrm{l}^{61}$ reported preservation of erectile function in $44 \%$ of patients, while Sveistrup et $\mathrm{al}^{65}$ stressed the issue that the use of hormonal therapy was associated with worse sexual symptoms. However, prostatic antigen decreased to values close to zero at the end of the treatment, thus leading consideration of clinical outcome as encouraging. No data on local control or OS are available.

To date, the study by Sveistrup et $\mathrm{al}^{65}$ is the one with the most comprehensive analysis of the symptoms before, during, and after the RT; however, the limitation of this work is the use of a questionnaire (Prostate Cancer Symptom Scale-PCSS scale) which makes it difficult to compare data with other experiences.

Pelvic lymph nodes, prostate, and seminal vesicles were treated by 5 authors ${ }^{62,64,66-68}$ using a single- to triple-arc technique, with doses ranging from $46.8 \mathrm{~Gy}$ for nodal coverage to $78 \mathrm{~Gy}$ for prostate irradiation. Almost all used IG as $\mathrm{kV}$ or CBCT, and $\sim 335$ patients were treated from 2012 to 2016. The conclusions were that VMAT-SIB is technically feasible and safe providing high target coverage and OAR sparing with acceptable GI and GU toxicity. Hegazy et al reported that $\sim 50 \%$ of patients (low and intermediate risk) with partial potency prior to RT retained functional potency on long-term follow-up (median 16 months; range: 14-32). ${ }^{67}$

Moreover, Hesselberg et $\mathrm{al}^{68}$ confirmed a favorable acute toxicity profile also in the case of treatment of large pelvic lymph node volumes with optimal dose coverage. Finally, $\mathrm{Ng}$ et $\mathrm{al}^{64}$ paid attention to a not trivial issue: as the population ages, the number of patients presenting with hip prostheses is expected to increase, and treatment planning for patients with metallic prosthesis composed of high $\mathrm{Z}$ materials posed challenges. In fact, hip prosthesis determines streaking and blurring artifacts in the CT data set which prevents accurate contour delineation and alters the image density values and moreover implies dose calculation uncertainties. ${ }^{70,71}$ In this context, $\mathrm{Ng}$ et $\mathrm{a}^{64}$ outlined that VMAT provides an elegant solution to deliver dose-escalated RT in patients with unilateral and bilateral hip replacements with minimal acute and late toxicities. Only Hegazy et al reported outcome data reporting 2- and 3-year biochemical recurrence-free survival of $90 \%$ and $72 \%$, respectively; the 3 - and 4 -year OS rates were $88 \%$ and $72 \%$, respectively (Table 4 ). ${ }^{67}$

More recently, clinical data on EC have been reported by Alongi et $\mathrm{al}^{72}$ and Macchia et $\mathrm{al}^{73}$ in 2 Phase I-II studies enrolling patients treated on pelvic lymph nodes and boosted on the vaginal cuff by VMAT-SIB dual-arc techniques. The 2-year local control rates were $98.5 \%$ and $100 \%$ in the 2 studies, with OS rates ranging from $94 \%$ and $96 \%$ (Table 4). Both authors concluded that VMAT is feasible and well tolerated in terms of acute as well as late toxicities. Indeed, while recognizing that vaginal brachytherapy represents the standard technique for vaginal vault irradiation (less dose to OAR, no organ motion, reduction of linear accelerator overload, and treatment time), the authors stated that VMAT-SIB not only represents a valid option in the case of lack of vaginal brachytherapy, but can be used in special conditions like unfavorable anatomy or patient refusal and/or claustrophobia. ${ }^{72,73}$ However, prolonged follow-up is needed to evaluate other clinical results including late toxicity and local control and survival. Additional issues such as secondary malignancy induction should also be examined, given the paucity of data relative to this type of risk for IMRT and VMAT techniques.

\section{Anorectal cancer: exclusive and neoadjuvant settings}

Exclusive or neoadjuvant concomitant chemoradiation is presently considered as the standard treatment option for 


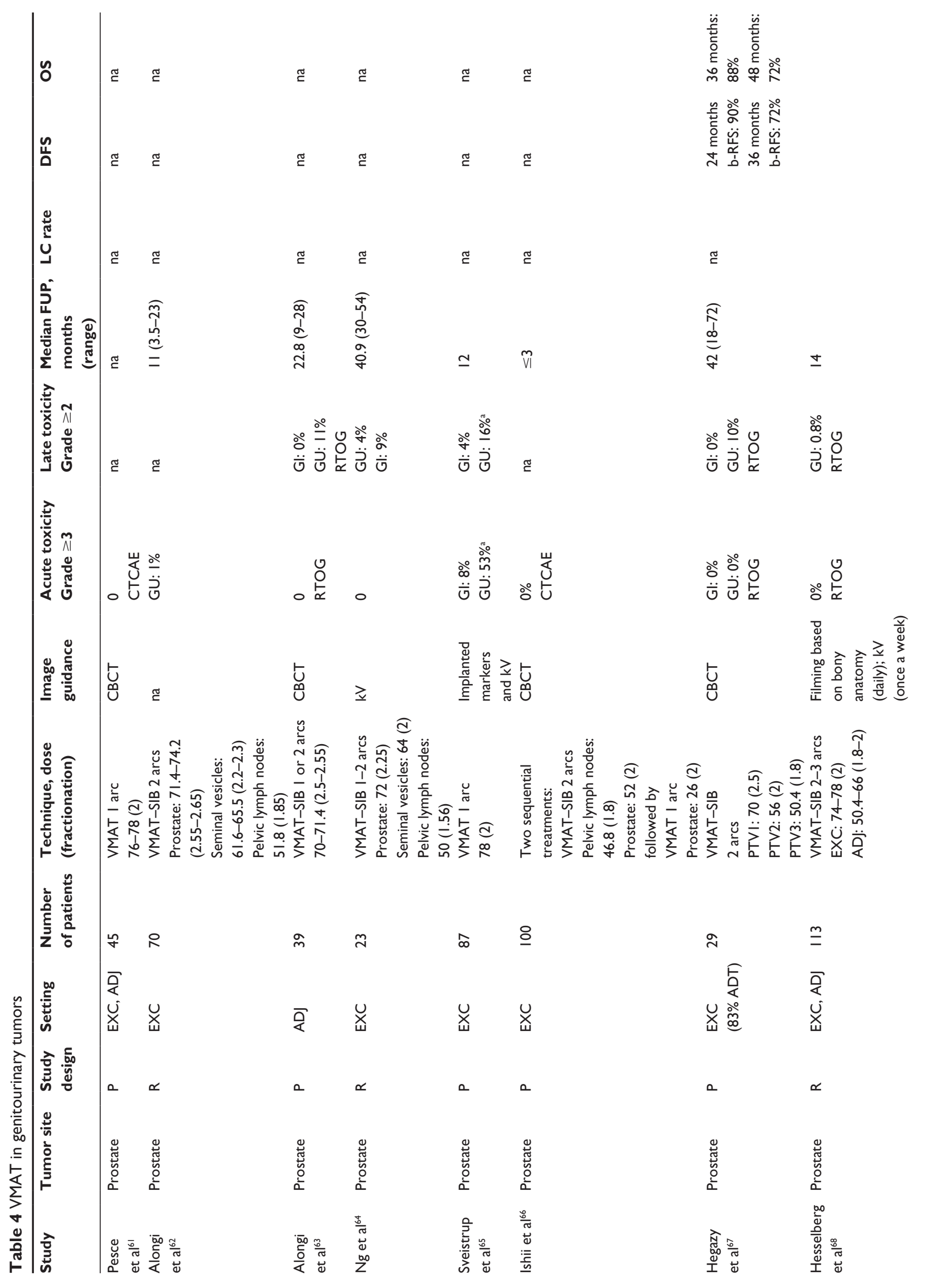




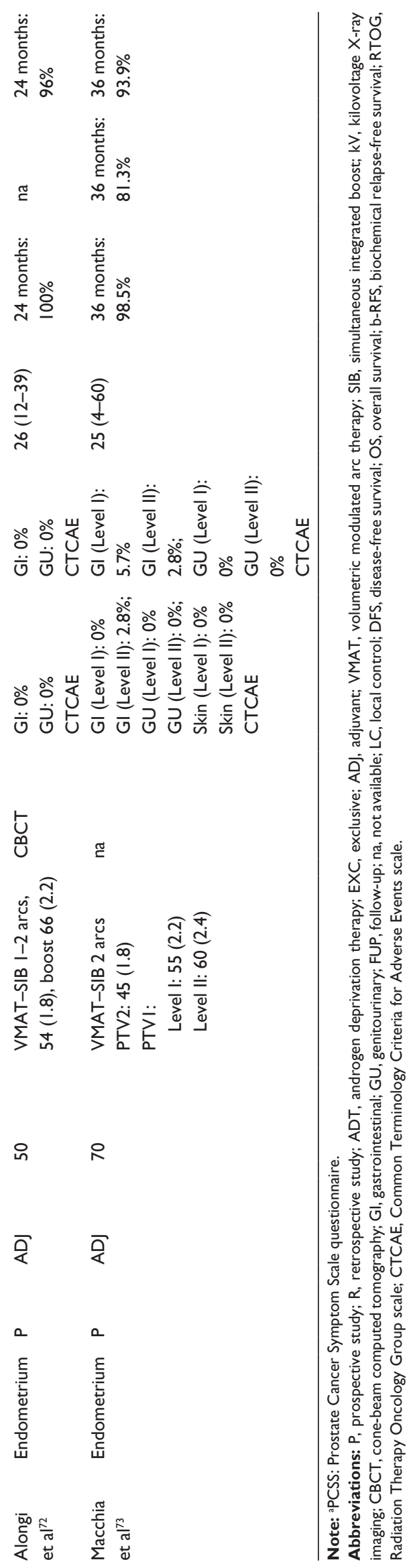

patients with anorectal cancer, providing consistent rates of locoregional control (LC), sphincter preservation, and OS. The acute toxicity profile linked to this approach, especially if a multidrug regimen is coupled to chemoradiation, refers particularly to skin, GI, and GU OARs and may potentially hinder the dose escalation to tumor volume, or stop treatment for a prolonged period, thereby reducing the radiobiological effectiveness of therapy. ${ }^{12,74}$ Seven clinical studies from 2010 to 2016 reported data on rectal or anal cancers treated by VMAT (Table 5). ${ }^{75-81}$ The rationale, also in this setting, is closely related to the higher OAR sparing, dose escalation possibility, and faster delivery compared to 3D-CRT or IMRT.

In particular, in the neoadjuvant setting of rectal cancer (RC), 3 studies accounting for 124 patients were included. . $^{75-77}$ Two of them, 1 prospective and 1 retrospective, compared VMAT technique to 3D-CRT with conventional doses (45/1.8 or $50.4 / 1.8$ Gy). ${ }^{75,76}$ Richetti et al ${ }^{75}$ prospectively treated $25 \mathrm{RC}$ patients by single arc, while Dröge et al ${ }^{76}$ retrospectively studied $\sim 81$ patients treated by a dual-arc technique; both authors concluded that VMAT improves conformality and reduces treatment times, with similar treatment outcomes. ${ }^{75,76}$ Moreover, in the larger study, ${ }^{76}$ a reduction of the high-grade acute and late toxicities was documented. On the other hand, in a Phase II study with concurrent multidrug chemotherapy (oxaliplatin plus capecitabine), Picardi et al ${ }^{77}$ first used the VMAT-SIB strategy to escalate doses on macroscopic disease up to $57.5 \mathrm{~Gy}$ (2.3 Gy/fraction), while delivering 45 Gy (1.8 Gy/fraction) to pelvic lymph nodes. The authors reported an excellent pathologic response rate (pT0-Tmic: $61.1 \%$ ) with quite encouraging outcomes also in terms of local control, DFS, and OS (Table 5). However, despite the use of the VMAT technique, $\sim 44 \%$ of patients had a non-negligible acute toxicity, hence they concluded that the implementation of IMRT and VMAT in RC is attractive and potentially facilitates dose escalation, but increases the risk of developing acute proctitis because the rectum inevitably receives high doses; therefore, the use of this scheme can be justified in clinical practice only in patients with advanced disease.

On the other hand, in the exclusive setting of anal cancer, 4 chemoradiation studies (1 Phase I and 3 retrospective ones accounting for 103 patients) were analyzed..$^{78-81}$ One of them ${ }^{80}$ investigated the irradiation of a single volume with either 2 full arcs or 4 half-arc VMAT technique and $6 \mathrm{MV}$ photon energies. The delivered dose of 50.4 Gy at 1.8 per fraction caused mostly severe skin toxicity $(29.4 \%)$, with low GI (11.8\%) and GU (5.9\%) severe toxicity. These figures are lower than the ones reported in 3D-CRT studies, for example, 


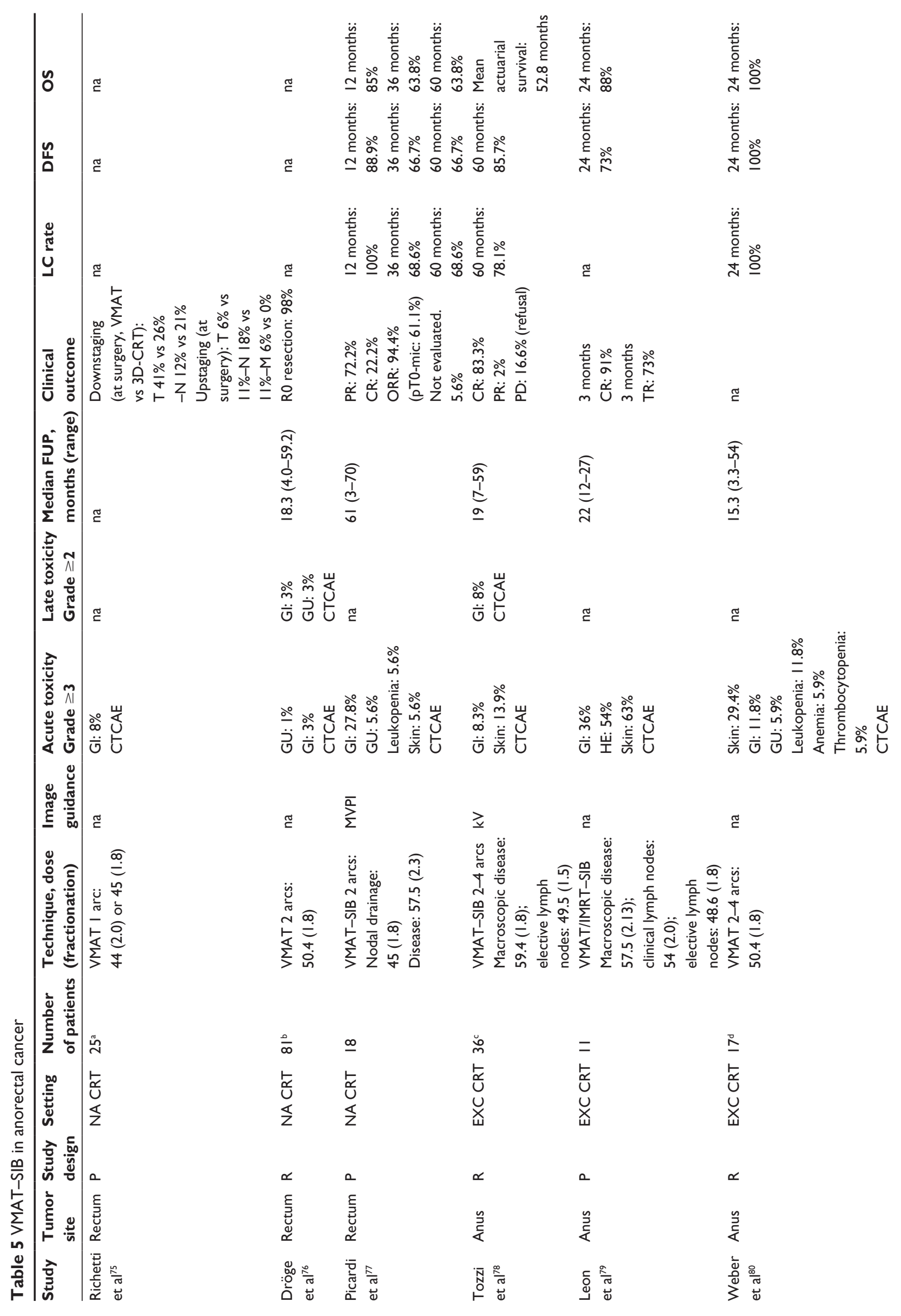




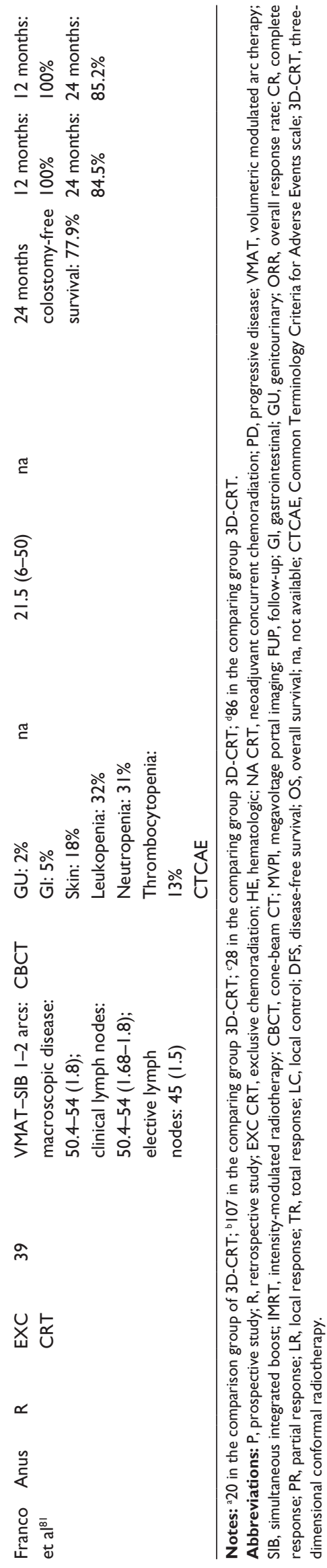

in Radiation Therapy Oncology Group 98-11 trial that reported a crude rate of acute Grade 3/Grade 4 toxicities of $48 \%$ for the skin and $35 \%$ for GI. ${ }^{82}$ One of the major issues that should be clarified for VMAT is whether single or multiple arcs should be applied to realize proper treatments. In our review, 3 studies employed a dual-arc SIB approach ${ }^{78-81}$ delivering a different daily dose to selected treatment volumes during the same treatment fraction. According to dosimetric results by Clivio et al, ${ }^{12}$ the double arcs can slightly improve the sparing of OAR guaranteeing more flexibility in dose modulation compared to single-arc treatments.

In 3 studies, ${ }^{78-80}$ elective lymph nodes received doses between 45 and $49.5 \mathrm{~Gy}$ (1.5 Gy/fraction); clinically detectable lymph nodes were treated with doses from 50.4 to $54 \mathrm{~Gy}$ (1.8-2 Gy/fraction), and macroscopic anal disease received doses from 50.4 to $59.4 \mathrm{~Gy}$ (1.8 Gy/fraction). Noteworthy, the SIB approach was able to shorten the overall treatment time with a consequent potential benefit on treatment outcomes.

The study reporting the most frequent toxicity was that by Leon et $\mathrm{al}^{79}$ who documented the rates of skin and GI toxicity in $63 \%$ and $36 \%$ of cases respectively, figures closer to 3D-CRT values than IMRT/VMAT. ${ }^{82}$ Besides the limits of the sample size $(\mathrm{N}=11)$, it is conceivable that these results may be ascribed to the higher total dose and, more probably, to the highest dose per fraction ( $2.13 \mathrm{~Gy}$ ) plus the addition of cetuximab that may have contributed to induce severe dermatitis given its peculiar skin toxicity properties. None of the other studies reported similar results, hence they cannot be justified given the poor clinical outcome (Table 5).

In conclusion, the available studies provided further lines of evidence supporting the implementation and use of VMAT-SIB on a routine basis for the treatment of cancer of the anal canal in combination with concurrent $\mathrm{CT}$ due to lower rates of acute organ toxicity and promising trends in LC and DFS.

\section{SBRT-VMAT (exclusive setting)}

In the "oligo-metastatic" setting, local approach may improve the outcome by keeping the burden of disease below the lethal threshold. However, most patients are unfit for surgery because of unfavorable tumor factors or poor general conditions. For these patients, a broad variety of alternative local therapeutic approaches are available besides conventional radiochemotherapy approaches. SBRT is a high-precision technique with tight planning margins and a sophisticated treatment plan allowing rapid dose fall-off away from the treatment area. It provides improved volume targeting and smaller irradiated volumes of normal tissue. On the other 
hand, VMAT is recognized to increase delivery efficiency and reduce the risk of intrafraction deviations in terms of both setup errors and organ motion; therefore, VMAT may represent a valuable technique for SBRT treatment.

Little evidence exists concerning the feasibility of SBRT-VMAT in different clinical settings; Table 6 reports isolated experiences in radical treatment of metastases or primary tumors at various sites. ${ }^{83-88} \mathrm{Six}$ studies (2 retrospective and 4 prospective Phase I-II trials) reported results on $\sim 284$ patients; in the majority of these studies, Image Guided by means of CBCT was used for daily treatment verification. ${ }^{83,85,87,88} \mathrm{~A}$ large variability of total dose and dose per fraction arose from the study analysis, probably due to the site of metastatic site as well as lack of literature about this issue. Of course, this variability limits any specific comparison, but it has to be recognized that the common denominator of the studies is the high rate of local control (range: $72 \%-94.7 \%$ ) and the low rate of acute toxicity recorded by the Common Terminology Criteria for Adverse Events scale.

A special consideration should tribute to the recent implementation of flattening filter-free (FFF) beams technology that increases the dose rate by removing flattening filter. ${ }^{89}$ The basis of the use of FFF beams for delivering SBRT doses is the potential possibility to deliver very high doses faster and more precisely, with a strong impact on time factor and, therefore, on intrafraction motion and total session treatment time. The FFF technology has been applied in Scorsetti et al and Franceschini et al trials, ${ }^{87,88}$ with the authors concluding that, in clinical practice, it could be potentially translated into less toxicity and subsequently in a better quality of life.

Among the reported studies, the trial by Deodato et a ${ }^{86}$ represents the only experience with stereotactic radiosurgery: with this technique, which is based on the delivery of a single high dose of radiation using high-precision technologies, favorable results in terms of response (95\%) and LC (2-year $\mathrm{LC}=72 \%$ ) were achieved with acceptable morbidity.

Similar findings were reported by Filippi et $\mathrm{a}^{90}$ who treated 90 lung lesions, of which 34 were managed by stereotactic radiosurgery; these results are not presented in Table 6 because it was not possible to retrieve the data about SBRT-VMAT from the whole series.

In conclusion, the possibility to easily integrate SBRTVMAT in the context of a systemic treatment due to intrinsic low toxicity and fast administration makes this approach very attractive. From a practical point of view, the introduction of VMAT for SBRT has resulted in a marked reduction of treatment time (especially by FFF beams technology), intrafraction uncertainties, costs related to highly complex

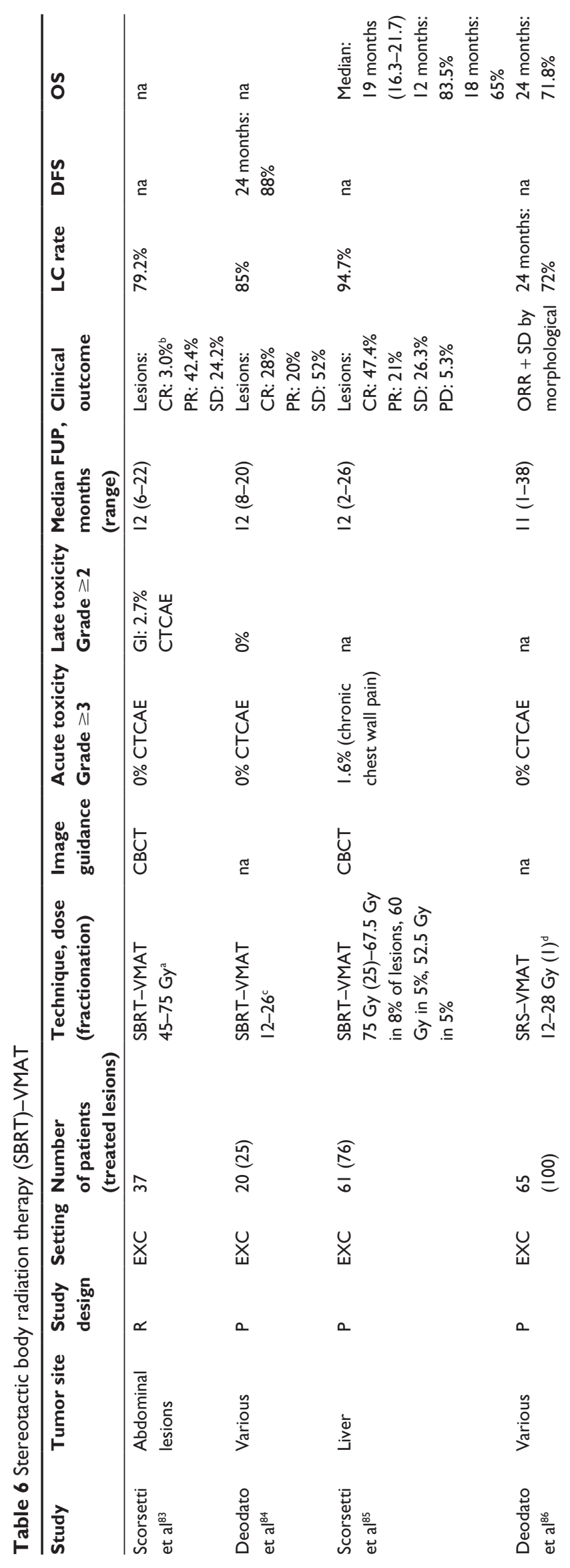




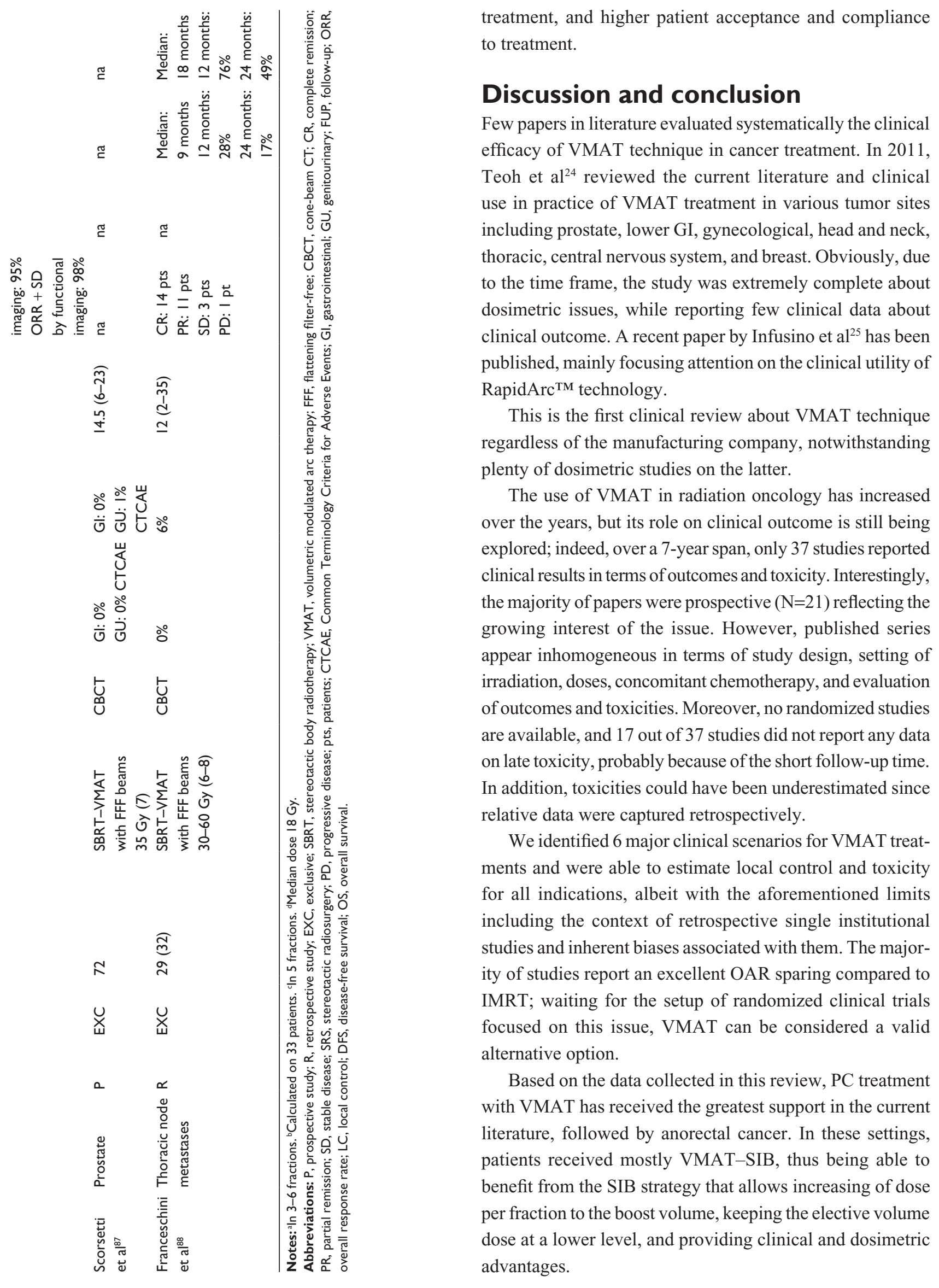


For all series, the therapeutic results appear encouraging, especially when large volumes need to be irradiated, and dual-arc SIB strategy is used, ${ }^{77}$ or high-sensitive structure sparing is required. ${ }^{32}$

In conclusion, the literature addressing the clinical appropriateness of VMAT is scarce. Current literature suggests that VMAT, especially when used as SIB or SBRT strategy, is an effective safe modality for all cancer types. Prospective studies with systematic data collection are needed for further understanding of the VMAT role in daily clinical use.

\section{Disclosure}

The authors report no conflicts of interest in this work.

\section{References}

1. Webb S. Intensity-Modulated Radiation Therapy. Bristol: Institute of Physics Publishing; 2000.

2. Mackie TR. History of tomotherapy. Phys Med Biol. 2006;51(13): R427-R453.

3. Yu CX. Intensity-modulated arc therapy with dynamic multileaf collimation: an alternative to tomotherapy. Phys Med Biol. 1995;40: 1435-1449.

4. Otto K. Volumetric modulated arc therapy: IMRT in a single gantry arc. Med Phys. 2008;35:310-317.

5. Palma DA, Verbakel WF, Otto K. New developments in arc radiation therapy: a review. Cancer Treat Rev. 2010;36(5):393-399.

6. Shaffer R, Nichol AM, Vollans E, et al. A comparison of volumetric modulated arc therapy and conventional intensity-modulated radiotherapy for frontal and temporal high-grade gliomas. Int J Radiat Oncol Biol Phys. 2010;76:1177-1184.

7. Verbakel WF, Cuijpers JP, Hoffmans D, Bieker M, Slotman BJ, Senan S. Volumetric intensity-modulated arc therapy vs. conventional IMRT in head-and-neck cancer: a comparative planning and dosimetric study. Int J Radiat Oncol Biol Phys. 2009;74:252-259.

8. Vanetti E, Clivio A, Nicolini G, et al. Volumetric modulated arc radiotherapy for carcinomas of the oro-pharynx, hypo-pharynx and larynx: a treatment planning comparison with fixed field IMRT. Radiother Oncol. 2009;92:111-117.

9. Cozzi L, Dinshaw KA, Shrivastava SK, et al. A treatment planning study comparing volumetric arc modulation with RapidArc and fixed field IMRT for cervix uteri radiotherapy. Radiother Oncol. 2008;89: 180-191.

10. Palma D, Vollans E, James K, et al. Volumetric modulated arc therapy for delivery of prostate radiotherapy: comparison with intensitymodulated radiotherapy and three-dimensional conformal radiotherapy. Int J Radiat Oncol Biol Phys. 2008;72:996-1001.

11. Kopp RW, Duff M, Catalfamo F, Shah D, Rajecki M, Ahmad K. VMAT vs. 7-field-IMRT: assessing the dosimetric parameters of prostate cancer treatment with a 292-patient sample. Med Dosim. 2011;36:365-372.

12. Clivio A, Fogliata A, Franzetti-Pellanda A, et al. Volumetric-modulated arc radiotherapy for carcinomas of the anal canal: a treatment planning comparison with fixed field IMRT. Radiother Oncol. 2009;92:118-124.

13. Rao M, Yang W, Chen F, et al. Comparison of Elekta VMAT with helical tomotherapy and fixed field IMRT: plan quality, delivery efficiency and accuracy. Med Phys. 2010;37:1350-1359.

14. Cilla S, Deodato F, Digesù $C$, et al. Assessing the feasibility of volumetric-modulated arc therapy using simultaneous integrated boost (SIB-VMAT): an analysis for complex head-neck, high-risk prostate and rectal cancer cases. Med Dosim. 2014;39:108-116.

15. Cilla S, Meluccio D, Fidanzio A, et al. Initial clinical experience with Epid-based in-vivo dosimetry for VMAT treatments of head-and-neck tumors. Phys Med. 2016;32:52-58.
16. Roa DE, Schiffner DC, Zhang J, et al. The use of RapidArc volumetricmodulated arc therapy to deliver stereotactic radiosurgery and stereotactic body radiotherapy to intracranial and extracranial targets. Med Dosim. 2012;37:257-264.

17. Wu QJ, Yoo S, Kirkpatrick JP, Thongphiew D, Yin FF. Volumetric arc intensity-modulated therapy for spine body radiotherapy: comparison with static intensity modulated treatment. Int J Radiat Oncol Biol Phys. 2009;75:1596-1604.

18. Audet C, Poffenbarger BA, Chang P, et al. Evaluation of volumetric modulated arc therapy for cranial radiosurgery using multiple noncoplanar arcs. Med Phys. 2011;38:5863-5872.

19. Scorsetti M, Mancosu P, Navarria P, et al. Stereotactic body radiation therapy (SBRT) for adrenal metastases: a feasibility study of advanced techniques with modulated photons and protons. Strahlenther Onkol. 2011;187:238-244.

20. Holt A, van Vliet-Vroegindeweij C, Mans A, Belderbos JS, Damen EM. Volumetric-modulated arc therapy for stereotactic body radiotherapy of lung tumors: a comparison with intensity-modulated radiotherapy techniques. Int J Radiat Oncol Biol Phys. 2011;81:1560-1567.

21. Macchia G, Deodato F, Cilla S, et al. Volumetric intensity modulated arc therapy for stereotactic body radiosurgery in oligometastatic breast and gynecological cancers: feasibility and clinical results. Oncol Rep. 2014;32:2237-2243.

22. Buwenge M, Cilla S, Guido A, et al. Individually optimized stereotactic radiotherapy for pancreatic head tumors: a planning feasibility study. Rep Pract Oncol Radiother. 2016;21:548-554.

23. Cilla S, Deodato F, Macchia G, et al. Linac-based extracranial radiosurgery with Elekta volumetric modulated arc therapy and an anatomybased treatment planning system: Feasibility and initial experience. Med Dosim. 2016;41:166-172.

24. Teoh M, Clark CH, Wood K, Whitaker S, Nisbet A. Volumetric modulated arc therapy: a review of current literature and clinical use in practice. Br J Radiol. 2011;84:967-996.

25. Infusino E. Clinical utility of RapidArcTM radiotherapy technology. Cancer Manag Res. 2015;7:345-356.

26. Linskey ME, Andrews DW, Asher AL, et al. The role of stereotactic radiosurgery in the management of patients with newly diagnosed brain metastases: a systematic review and evidence based clinical practice guideline. J Neurooncol. 2010;96:45-68.

27. Tsao MN, Rades D, Wirth A, et al. Radiotherapeutic and surgical management for newly diagnosed brain metastasis(es): an American Society for Radiation Oncology evidence-based guideline. Pract Radiat Oncol. 2012;2:210-225.

28. McDuff SG, Taich ZJ, Lawson JD, et al. Neurocognitive assessment following whole brain radiation therapy and radiosurgery for patients with cerebral metastases. J Neurol Neurosurg Psychiatry. 2013;84: 1384-1391.

29. Wen PY, Black PM, Loeffler JS. Metastatic brain cancer. In: DeVita V, Hellman S, Rosenberg SA, editors. Cancer: Principles and Practice of Oncology. 6th ed. Philadelphia, PA, USA: Lippincott, Williams, \& Wilkins; 2001:2655-2670.

30. Weber DC, Caparrotti F, Laouiti M, Malek K. Simultaneous in-field boost for patients with 1 to 4 brain metastasis/es treated with volumetric modulated arc therapy: a prospective study on quality-of-life. Radiat Oncol. 2011;6:79.

31. Awad R, Fogarty G, Hong A, et al. Hippocampal avoidance with volumetric modulated arc therapy in melanoma brain metastases-the first Australian experience. Radiat Oncol. 2013;8:62.

32. Nichol A, Ma R, Hsu F, et al. Volumetric radiosurgery for 1 to 10 brain metastases: a multicenter, single-arm, phase 2 study. Int J Radiat Oncol Biol Phys. 2016;94:312-321.

33. Tsai PF, Yang CC, Chuang CC, et al. Hippocampal dosimetry correlates with the change in neurocognitive function after hippocampal sparing during whole brain radiotherapy: a prospective study. Radiat Oncol. 2015;10:253.

34. Ferlay J, Autier P, Boniol M, Heanue M, Colombet M, Boyle P. Estimates of the cancer incidence and mortality in Europe in 2006. Ann Oncol. 2007;18:581-592. 
35. Forastiere AA, Goepfert H, Maor M, et al. Concurrent chemotherapy and radiotherapy for organ preservation in advanced laryngeal cancer. N Engl J Med. 2003;349:2091-2098.

36. Corvò R. Evidence-based radiation oncology in head and neck squamous cell carcinoma. Radiother Oncol. 2007;85(1):156-170.

37. Eisbruch A, ten Haken RK, Kim HM, Marsh LH, Ship JA. Dose volume and function relationships in parotid glands following conformal and intensity modulated irradiation of head and neck cancer. Int J Radiat Oncol Biol Phys. 1999;45:577-587.

38. Gregoire V, Maingon P. Intensity modulated radiation therapy in head and neck squamous cell carcinoma: state of art and future challenges. Cancer Radiother. 2005;9:42-50.

39. Dirix P, Nuyts S. Evidence-based organ-sparing radiotherapy in head and neck cancer. Lancet Oncol. 2010;11:85-91.

40. Nutting CM, Morden JP, Harrington KJ, et al; PARSPORT trial management group. Parotid-sparing intensity modulated versus conventional radiotherapy in head and neck cancer (PARSPORT): a phase 3 multicentre randomised controlled trial. Lancet Oncol. 2011;12: 127-136.

41. Hall EJ. Intensity-modulated radiation therapy, protons, and the risk of second cancers. Int J Radiat Oncol Biol Phys. 2006;65:1-7.

42. Fung-Kee-Fung SD, Hackett R, Hales L, Warren G, Singh AK. A prospective trial of volumetric intensity-modulated arc therapy vs conventional intensity modulated radiation therapy in advanced head and neck cancer. World J Clin Oncol. 2012;3:57-62.

43. Scorsetti M, Fogliata A, Castiglioni S, et al. Early clinical experience with volumetric modulated arc therapy in head and neck cancer patients. Radiat Oncol. 2010;5:93.

44. Doornaert P, Verbakel WF, Bieker M, Slotman BJ, Senan S. RapidArc planning and delivery in patients with locally advanced head-and-neck cancer undergoing chemoradiotherapy. Int J Radiat Oncol Biol Phys. 2011;79:429-435.

45. Smet S, Lambrecht M, Vanstraelen B, Nuyts S. Clinical and dosimetric evaluation of RapidArc versus standard sliding window IMRT in the treatment of head and neck cancer. Strahlenther Onkol. 2015;191: 43-50.

46. Moncharmont C, Vallard A, Mengue Ndong S, et al. Real-life assessment of volumetric modulated arc therapy (VMAT) toxicity in head and neck squamous cell carcinoma (HNSCC) treatment. Acta Otolaryngol. 2016;136:181-188.

47. Ozdemir S, Akin M, Coban Y, Yildirim C, Uzel O. Acute toxicity in nasopharyngeal carcinoma patients treated with IMRT/VMAT. Asian Pac J Cancer Prev. 2015;16:1897-1900.

48. Guo R, Tang LL, Mao YP, et al. Clinical Outcomes of Volume-Modulated Arc Therapy in 205 Patients with Nasopharyngeal Carcinoma: An Analysis of Survival and Treatment Toxicities. PLoS One. 2015; 10:e0129679.

49. Scorsetti M, Navarria P, Mancosu P, et al. Large volume unresectable locally advanced non-small cell lung cancer: acute toxicity and initial outcome results with rapid arc. Radiat Oncol. 2010;5:94.

50. Kimura T, Doi Y, Nakashima T, et al. Clinical experience of volumetric modulated arc therapy for malignant pleural mesothelioma after extrapleural pneumonectomy. J Radiat Res. 2015;56:315-324.

51. Kim YJ, Kim K, Lee R, et al. Two-year follow-up of volumetricmodulated arc therapy for treating internal mammary nodes in locally advanced breast cancer. Anticancer Res. 2016;36:4847-4851.

52. De Rose F, Fogliata A, Franceschini D, et al. Phase II trial of hypofractionated VMAT-based treatment for early stage breast cancer: 2-year toxicity and clinical results. Radiat Oncol. 2016;11:120.

53. Popescu CC, Beckham WA, Patenaude VV, Olivotto IA, Vlachaki MT. Simultaneous couch and gantry dynamic arc rotation (CG-Darc) in the treatment of breast cancer with accelerated partial breast irradiation (APBI): a feasibility study. J Appl Clin Med Phys. 2013;14: 4035.

54. Scorsetti M, Bignardi M, Clivio A, et al. Volumetric modulation arc radiotherapy compared with static gantry intensity modulated radiotherapy for malignant pleural mesothelioma tumor: a feasibility study. Int J Radiat Oncol Biol Phys. 2010;77:942-949.
55. Krayenbuehl J, Riestere O, Graydon S, Dimmerling P, Kloeck S, Ciernik IF. Intensity-modulated radiotherapy and volumetric-modulated arc therapy for malignant pleural mesothelioma after extrapleural pleuropneumonectomy. J Appl Clin Med Phys. 2013;14:1-10.

56. Popescu CC, Olivotto IA, Beckham WA, et al. Volumetric modulated arc therapy improves dosimetry and reduces treatment time compared to conventional intensity-modulated radiotherapy for locoregional radiotherapy of left-sided breast cancer and internal mammary nodes. Int J Radiat Oncol Biol Phys. 2010;76:287-295.

57. Scorsetti M, Alongi F, Fogliata A, et al. Phase I-II study of hypofractionated simultaneous integrated boost using volumetric modulated arc therapy for adjuvant radiation therapy in breast cancer patients: a report of feasibility and early toxicity results in the first 50 treatments. Radiat Oncol. 2012;7:145

58. Yoo S, Wu QJ, Lee WR, Yin FF. Radiotherapy treatment plans with RapidArc for prostate cancer involving seminal vesicles and lymph nodes. Int J Radiat Oncol Biol Phys. 2010;76:935-942.

59. Zhang P, Happersett L, Hunt M, Jackson A, Zelefsky M, Mageras G. Volumetric modulated arc therapy: planning and evaluation for prostate cancer cases. Int J Radiat Oncol Biol Phys. 2010;76: $1456-1462$

60. Myrehaug S, Chan G, Craig T, et al. A treatment planning and acute toxicity comparison of two pelvic nodal volume delineation techniques and delivery comparison of intensity-modulated radiotherapy versus volumetric modulated arc therapy for hypofractionated high-risk prostate cancer radiotherapy. Int J Radiat Oncol Biol Phys. 2012;82: e657-e662.

61. Pesce GA, Clivio A, Cozzi L, et al. Early clinical experience of radiotherapy of prostate cancer with volumetric modulated arc therapy. Radiat Oncol. 2010;5:54.

62. Alongi F, Fogliata A, Navarria P, et al. Moderate hypofractionation and simultaneous integrated boost with volumetric modulated arc therapy (RapidArc) for prostate cancer. Report of feasibility and acute toxicity. Strahlenther Onkol. 2012;188:990-996.

63. Alongi F, Cozzi L, Fogliata A, et al. Hypofractionation with VMAT versus 3DCRT in post-operative patients with prostate cancer. Anticancer Res. 2013;33:4537-4543.

64. Ng WL, Brunt J, Temple S, et al. Volumetric modulated arc therapy in prostate cancer patients with metallic hip prostheses in a UK centre. Rep Pract Oncol Radiother. 2015;20:273-277.

65. Sveistrup J, Widmark A, Fransson P, et al. Prospective assessment of urinary, gastrointestinal and sexual symptoms before, during and after image-guided volumetric modulated arc therapy for prostate cancer. Scand J Urol. 2015;49:58-69.

66. Ishii K, Ogino R, Hosokawa Y, et al. Whole-pelvic volumetric-modulated arc therapy for high-risk prostate cancer: treatment planning and acute toxicity. J Radiat Res. 2015;56:141-150.

67. Hegazy MW, Mahmood RI, Al Otaibi MF, Khalil EM. Hypofractionated volumetric modulated arc radiotherapy with simultaneous elective nodal irradiation is feasible in prostate cancer patients: a single institution experience. J Egypt Natl Canc Inst. 2016;28:101-110.

68. Hesselberg G, Fogarty G, Haydu L, Dougheney N, Stricker P Volumetric modulated arc therapy of the pelvic lymph nodes to the aortic bifurcation in higher risk prostate cancer: early toxicity outcomes. Biomed Res Int. 2015;2015:696439.

69. Langen KM, Willoughby TR, Meeks SL, et al. Observation on realtime prostate gland motion using electromagnetic tracking. Int J Radiat Oncol Biol Phys. 2008;71:1084-1090.

70. Eng TY. Dose attenuation through a titanium alloy hip prosthesis. Med Dosim. 2000;25:7-8.

71. Hazuka MB, Stroud DN, Adams J, Ibbott GS, Kinzie JJ. Prostatic thermoluminescent dosimeter analysis in a patient treated with $18 \mathrm{MV}$ $\mathrm{X}$ rays through a prosthetic hip. Int J Radiat Oncol Biol Phys. 1993; 25:339-343.

72. Alongi F, Mazzola R, Ricchetti F, et al. Volumetric-modulated arc therapy with vaginal cuff simultaneous integrated boost as an alternative to brachytherapy in adjuvant irradiation for endometrial cancer: a prospective study. Anticancer Res. 2015;35:2149-2155. 
73. Macchia G, Cilla S, Deodato F, et al. Simultaneous integrated boost volumetric modulated arc therapy in the postoperative treatment of high-risk to intermediate-risk endometrial cancer: results of ADA II phase 1-2 trial. Int J Radiat Oncol Biol Phys. 2016;96:606-613.

74. Hong TS, Moughan J, Garofalo MC, et al. A phase 2 study of preoperative chemoradiation therapy using intensity modulated radiation therapy in combination with capecitabine and oxaliplatin for patients with locally advanced rectal cancer. Int J Radiat Oncol Biol Phys. 2015;93:29-36.

75. Richetti A, Fogliata A, Clivio A, et al. Neo-adjuvant chemo-radiation of rectal cancer with volumetric modulated arc therapy: summary of technical and dosimetric features and early clinical experience. Radiat Oncol. 2010;5:14.

76. Dröge LH, Weber HE, Guhlich M, et al. Reduced toxicity in the treatment of locally advanced rectal cancer: a comparison of volumetric modulated arc therapy and 3D conformal radiotherapy. BMC Cancer. 2015;15:750.

77. Picardi V, Macchia G, Guido A, et al. Preoperative chemoradiation with VMAT-SIB in rectal cancer: a phase II study. Clin Colorectal Cancer. 2017;16(1):16-22.

78. Tozzi A, Cozzi L, Iftode C, et al. Radiation therapy of anal canal cancer: from conformal therapy to volumetric arc therapy. BMC Cancer. 2014;14:833.

79. Leon O, Guren MG, Radu C, Gunnlaugsson A, Johnsson A. Phase I study of cetuximab in combination with 5-fluorouracil, mitomycin C and radiotherapy in patients with locally advanced anal cancer. Eur $J$ Cancer. 2015;51:2740-2746.

80. Weber HE, Dröge LH, Hennies S, Herrmann MK, Gaedcke J, Wolff HA. Volumetric intensity-modulated arc therapy vs. 3-dimensional conformal radiotherapy for primary chemoradiotherapy of anal carcinoma. Strahlentherapie und Onkol. 2015;191:827-834.

81. Franco P, Arcadipane F, Ragona R, et al. Volumetric modulated arc therapy (VMAT) in the combined modality treatment of anal cancer patients. Br J Radiol. 2016;17:20160832.
82. Ajani JA, Winter KA, Gunderson LL, et al. Fluorouracil, mitomycin, and radiotherapy vs fluorouracil, cisplatin, and radiotherapy for carcinoma of the anal canal: a randomized controlled trial. JAMA. 2008;299:1914-1921.

83. Scorsetti M, Bignardi M, Alongi F, et al. Stereotactic body radiation therapy for abdominal targets using volumetric intensity modulated arc therapy with RapidArc: feasibility and clinical preliminary results. Acta Oncol. 2011;50(4):528-538.

84. Deodato F, Cilla S, Macchia G, et al. Extracranial radiosurgery with volumetric modulated arc therapy: Feasibility evaluation of a phase I trial. Oncol Lett. 2013;5:1889-1896.

85. Scorsetti M, Arcangeli S, Tozzi A, et al. Is stereotactic body radiation therapy an attractive option for unresectable liver metastases? A preliminary report from a phase 2 trial. Int J Radiat Oncol Biol Phys. 2013;86:336-342.

86. Deodato F, Cilla S, Macchia G, et al. Stereotactic radiosurgery (SRS) with volumetric modulated arc therapy (VMAT): interim results of a multi-arm phase I trial (DESTROY-2). Clin Oncol ( $R$ Coll Radiol). 2014;26:748-756.

87. Scorsetti M, Alongi F, Clerici E, et al. Stereotactic body radiotherapy with flattening filter-free beams for prostate cancer: assessment of patient-reported quality of life. J Cancer Res Clin Oncol. 2014; 140:1795-1800.

88. Franceschini D, De Rose F, Fogliata A, et al. Volumetric modulated arc therapy for thoracic node metastases: a safe and effective treatment for a neglected disease. Oncotarget. 2016;7:53321-53329.

89. Dang TM, Peters MJ, Hickey B, Semciw A. Efficacy of flattening-filterfree beam in stereotactic body radiation therapy planning and treatment: a systematic review with meta-analysis. J Med Imaging Radiat Oncol. 2017;61(13):379-387.

90. Filippi AR, Badellino S, Guarneri A, et al. Outcomes of single fraction stereotactic ablative radiotherapy for lung metastases. Technol Cancer Res Treat. 2014;13:37-45.
OncoTargets and Therapy

\section{Publish your work in this journal}

OncoTargets and Therapy is an international, peer-reviewed, open access journal focusing on the pathological basis of all cancers, potential targets for therapy and treatment protocols employed to improve the management of cancer patients. The journal also focuses on the impact of management programs and new therapeutic agents and protocols on

\section{Dovepress}

patient perspectives such as quality of life, adherence and satisfaction The manuscript management system is completely online and includes a very quick and fair peer-review system, which is all easy to use. Visit http://www.dovepress.com/testimonials.php to read real quotes from published authors. 